\title{
Robot Leg Motion in a Planarized-SOI, 2 Poly Process
}

\author{
Seth Hollar ${ }^{1}$, Anita Flynn ${ }^{2}$, Sarah Bergbreiter ${ }^{1}$, K.S.J.Pister ${ }^{1}$ \\ ${ }^{1}$ Berkeley Sensor and Actuator Center, University of California, Berkeley 94720 \\ ${ }^{2}$ Micropropulsion Corporation, Berkeley, CA 94704
}

\begin{abstract}
With the ultimate goal of creating autonomous microrobots, we have developed a new five-mask process that combines two polysilicon structural layers with $35 \mu \mathrm{m}$ thick SOI structures and a backside substrate etch. The polysilicon layers provide 3D hinged structures, high compliance structures, and electrical wiring. The SOI structural layer yields much stronger structures and large-force actuators. On an $8 \mathrm{~mm} \mathrm{x}$ $3 \mathrm{~mm}$ robot, we have demonstrated polysilicon hinges with SOI electrostatic inchworm motors. This combination allowed us to demonstrate $200 \mu \mathrm{m}$ out-of-plane motion of a hinged SOI robot leg. Using an off-chip controller, the robot leg was actuated through almost $30^{\circ}$. In addition, we have completed static friction tests of polysilicon flaps to more accurately model the frictional forces of the linkages.
\end{abstract}

\section{INTRODUCTION}

Until recently, one of the greatest challenges in designing autonomous microrobots has been finding actuators that are efficient and capable of large forces and displacements. Shimoyama, et al. have suggested providing external actuation and power through vibratory or magnetic fields [1,2]. Kladitis [3] and Ebefors [4] have both demonstrated walking microrobots using electro-thermal actuation and off-chip power supplies. While thermal actuators achieve the large forces and displacements necessary for a microrobot, the large power requirements prevent these robots from becoming autonomous.

In an effort to design lower power actuators, Yeh et al. developed an electrostatic inchworm motor that could be fabricated with a single mask SOI process [5]. Inchworm motors combine large forces from gap closing actuators (GCAs) with large displacements by continually repeating small steps.

A second challenge of microrobots has been to combine the aforementioned actuators with compliant structures to create out-of-plane motion. Yeh suggested various hinged structures which could be folded to create an articulated microrobot [6]. However, these linkages were not easily combined with a means of low-power actuation.

To this end, we have developed a process in which electrostatic inchworm motors in thick SOI can be fabricated with thinner polysilicon structures on top. This process combined with solar cells and digital electronics provides us with a basis for designing autonomous walking microrobots. Figure 1 shows a conceptual rendering of our current design. A CMOS controller chip generates signals to drive the motors. A chip with solar cells and high voltage buffers provides power and converts the low voltage CMOS signals to the 30 volts needed to drive the actuators.

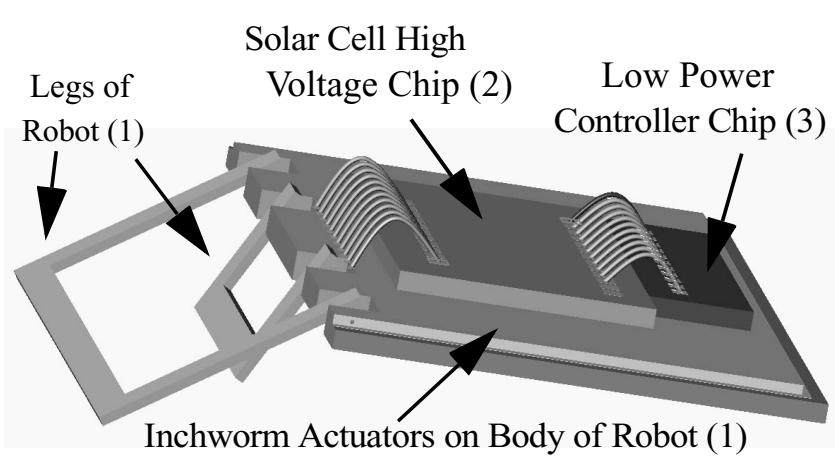

Figure 1. Computer modeled rendering of walking robot. The 3-chip hybrid is composed of 1) Legs and Motor process, 2) Solar Cells and High Voltage process and 3) Low Power CMOS process.

\section{FABRICATION PROCESS}

We designed a new process that combines low power, high force actuators in SOI with hinged structures and linkages from traditional polysilicon micromachining. An SEM of a typical hinge is shown in Figure 2 and a flow diagram of the five mask process is shown in Figure 3.

We started with an SOI wafer that had a device layer thickness of $20-40 \mu \mathrm{m}$ (Figure 3a). The top layer was patterned and etched using the Bosch ASE process (Figure 3b).

A key challenge of the process was adding structural polysilicon after the primary SOI etch. This was accomplished using Yasseen's method of thick glass planarization. Following [7], we spun on a glass slurry (Figure 3c), burnt out the organic (Figure 3d), and reflowed the glass at $890 \mathrm{C}$, where

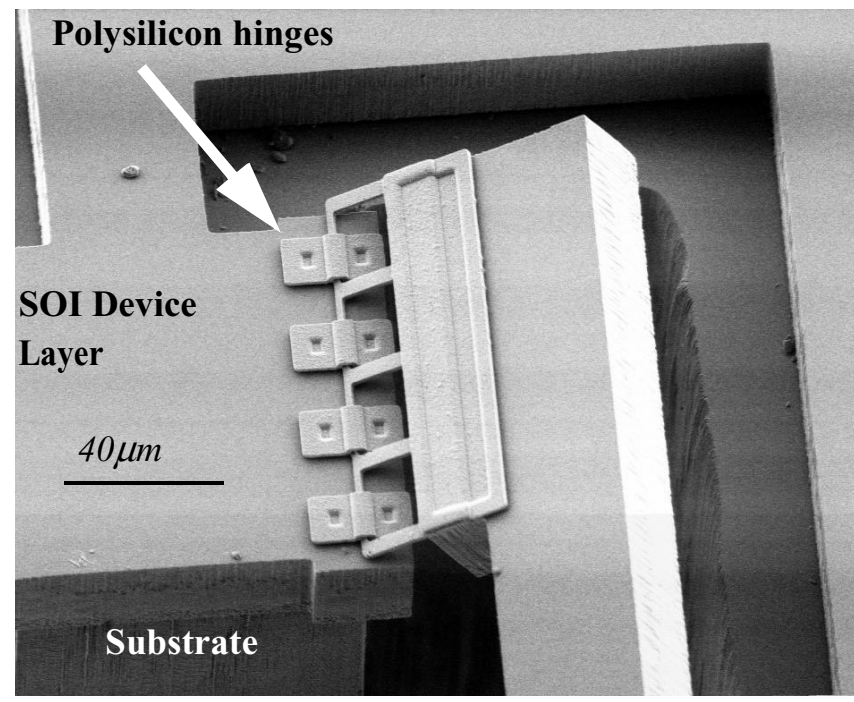

Figure 2. Polysilicon hinges attached to SOI device layer. The substrate is also seen in this SEM. 
(a)

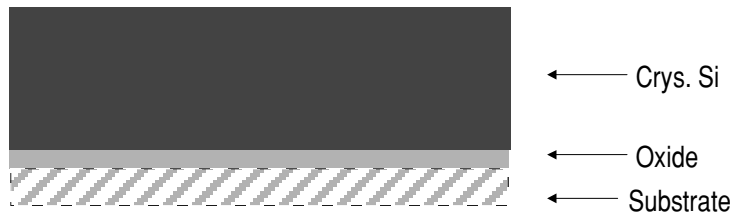

(b)

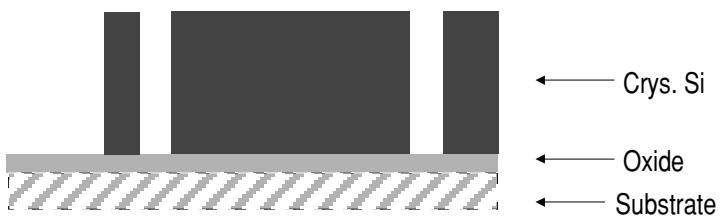

(c)

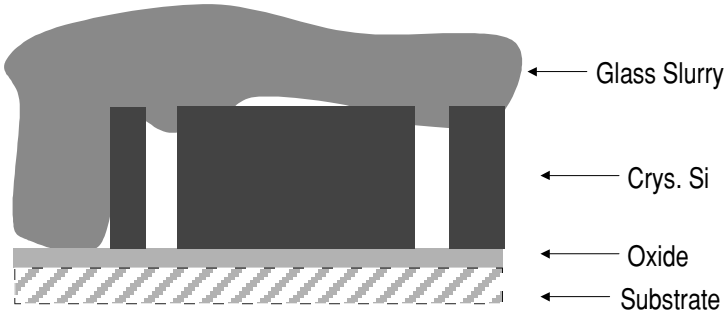

(d)

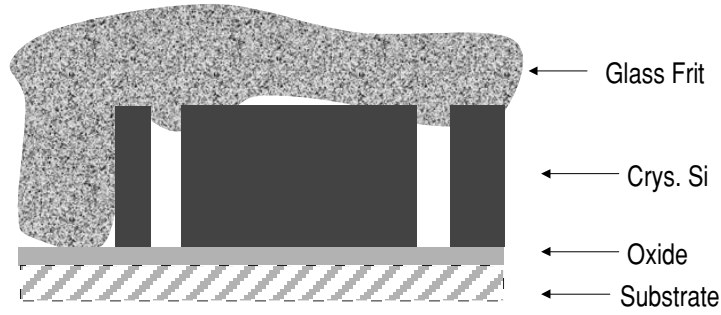

(e)

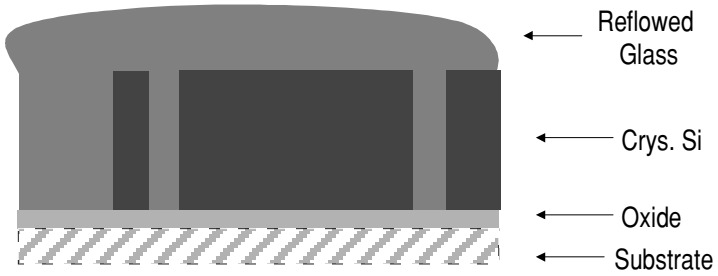

(f)

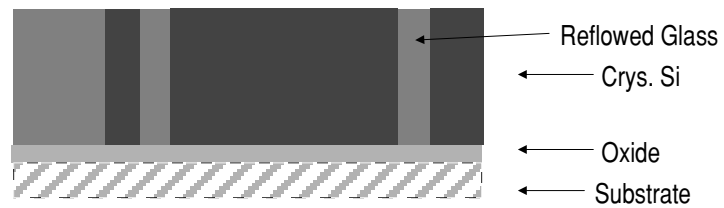

(g)

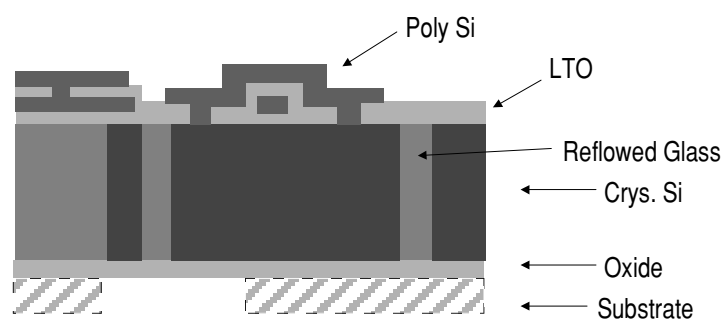

(h)
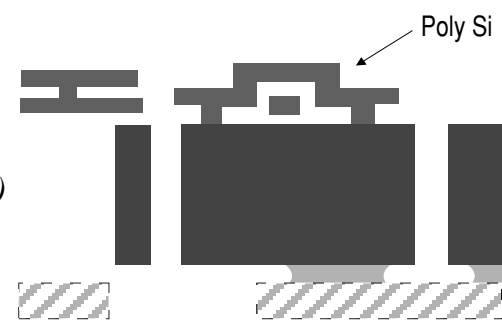

$\longleftarrow$ Crys. Si

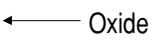

$\longleftarrow$ Substrate

Figure 3. Process Flow.

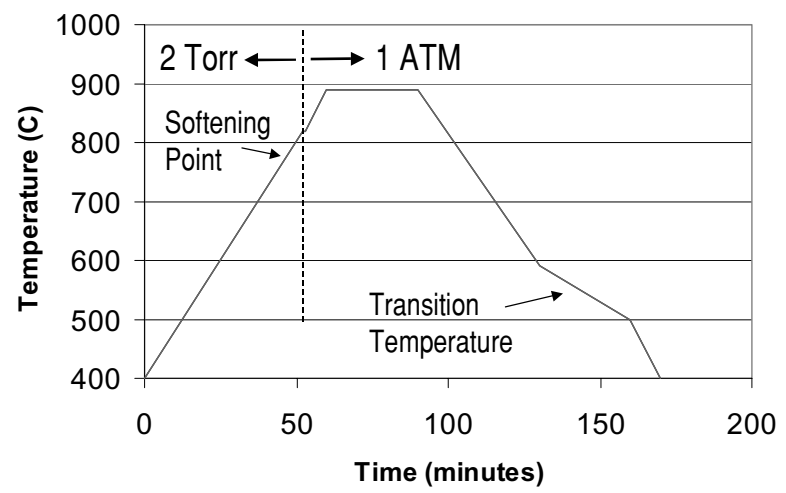

Figure 4. Firing profile of glass. The glass was heated in an oxygen ambient at 2 Torr to allow the glass to flow into deep trenches. Any remaining bubbles were then minimized during the pressurization stage from 2 Torr to 1 ATM.

the frit coalesced to form a single glass network (Figure 3e). The wafers were fired in a low pressure furnace to minimize the occurrence of bubbles. However, we found that simply firing in low pressure did not completely remove bubbles. As a deviation from [7], we heated the glass to just below the firing temperature in an oxygen ambient at 2 Torr to allow the glass to flow into deep trenches. Any remaining bubbles were then minimized during the pressurization stage from 2 Torr to 1 ATM. The firing profile is shown in Figure 4. Finally, the glass was chemically mechanically polished (CMP) to the SOI/ glass interface (Figure 3f) to provide a planarized surface.

After planarization, wafers underwent standard polysilicon micromachining. Three masks were used to define 2 structural polysilicon layers (fine-line lithography: $2 \mu \mathrm{m}$ ). $0.6 \mu \mathrm{m}$ of PSG was first deposited on the planarized SOI surface, followed by a $2 \mu \mathrm{m}$ thick layer of patterned polysilicon. After another $0.6 \mu \mathrm{m}$ PSG deposition, the wafer was patterned with a contact mask used for anchoring the following polysilicon layer. Next, $2 \mu \mathrm{m}$ of the second and final polysilicon layer was deposited. Finally, a backside substrate etch was performed using the same Bosch ASE process as before (Figure 3g).

For the release, a solution of $\mathrm{HCl}, \mathrm{HF}$ and $\mathrm{H}_{2} \mathrm{O}$ was used in a timed etch on the planarized glass, buried thermal oxide, and PSG. The goal was to etch long enough to remove the PSG and reflowed glass, but not so long as to remove all of the bur-

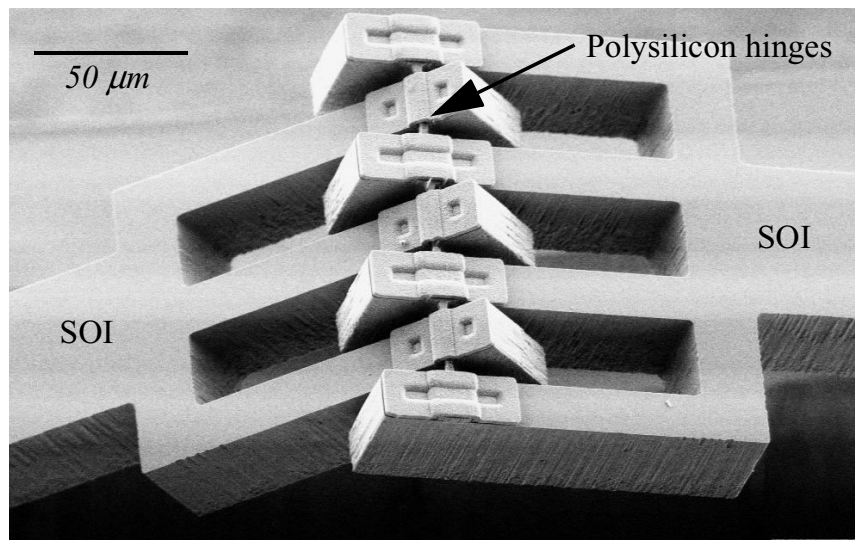

Figure 5. Polysilicon hinge structure with interdigitated device layer SOI. 
ied oxide. The reflowed glass etches approximately 30 times faster than the buried oxide, so tight tolerances in the undercut of the thermal oxide were maintained. Narrow SOI blocks were fully undercut while wide SOI blocks were still anchored to the substrate via the thermal oxide (Figure $3 h$ ).

An unfortunate by-product of the release was that alumina particles were scattered across the wafer. Since the glass frit was created at Ferro Corporation using an alumina ball mill for an extended period of time, alumina flakes on the order of $0.5 \mu \mathrm{m}$ were present in the glass mixture. To remove the alumina particles, the devices were placed in a heated bath $\left(50^{\circ} \mathrm{C}\right)$ of $\mathrm{NH}_{4} \mathrm{OH}$ and $\mathrm{H}_{2} \mathrm{O}_{2}$. Critical point drying was performed to avoid damage from water surface tension.

\section{ROBOT LEG DESIGN}

Using the five-mask process described above, we designed an $8 \mathrm{~mm} \times 3 \mathrm{~mm}$ robot that uses inchworm motors and hinged SOI legs. We used pin hinges (Figures 2 and 5) in a 1 DOF leg whose linkages are depicted in the kinematically equivalent 2D diagram in Figure 6. The robot design incorporates a number of design advantages from the process and remedies some problems associated with Yeh's original inchworm motor.

Since both the SOI and polysilicon act as conductors, the motor wiring is not constrained to any particular layer. This allowed us to draw connections from the motors into a set of bond pads in the back of the robot. An example of a polysilicon jumper is shown in Figure 7. However, wiring over long distances was problematic. Since both the SOI and polysilicon are susceptible to pull-in after the PSG and thermal oxide lay-

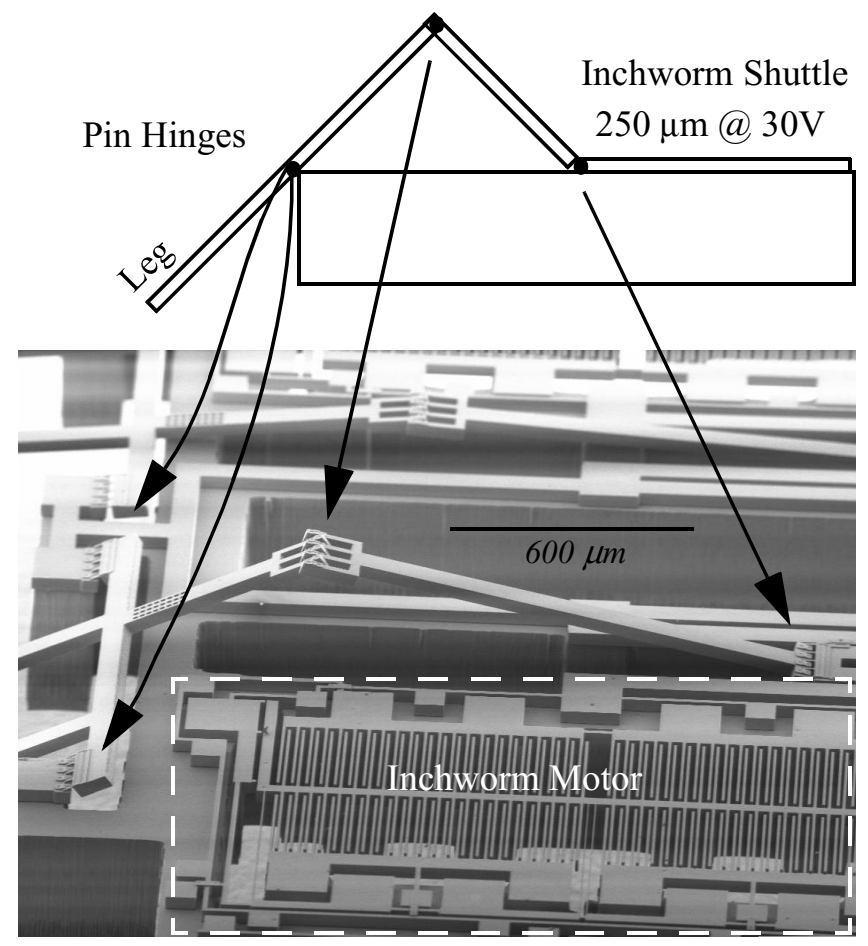

Figure 6. Kinematic diagram of robot leg and SEM of linkage structure. Arrows are used to show the position of hinges relative to the diagram. In the lower right hand corner of the SEM, one of the drive GCAs of the inchworm motor is visible.

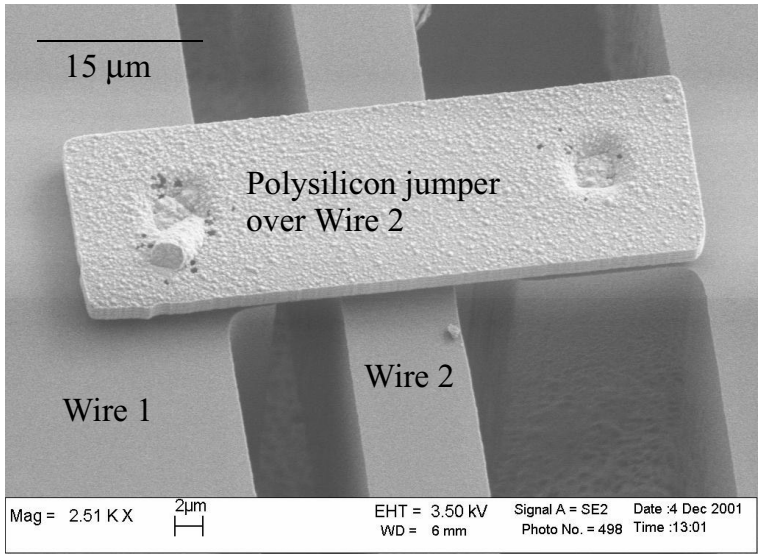

Figure 7. A polysilicon jumper bridges Wire 2.

ers are removed, SOI anchors were placed periodically along the wire. These anchors provided enough mechanical stiffness to withstand electrostatic forces between the SOI wiring and robot substrate.

The improved inchworm motors also utilized flaps to constrain the motion of the shuttle to the plane of the device. As seen in Figure 8, the flap attached to the shuttle on the left prevents the shuttle from falling through the wafer, and the flap over the shuttle on the right prevents the shuttle from popping up out-of-plane. With the inclusion of flaps, the shuttle is no longer constrained by parallel flexures as in [5].

The backside etch yielded several advantages over the original inchworm motor process and for the robot in general. Removing the substrate underneath movable structures in the inchworm motors prevented substrate stiction and reduced the chance particulate matter would get stuck between the gap closing actuators. Additionally, backside removal provided a lightened skeletal frame, which reduced the amount of force required from the motors.

Preset structures can be set so as to maximize mechanical advantage during the initial actuation. Without preset structures, the out-of-plane moment arm is limited to the thickness of the SOI. As shown in Figure 9, a preset structure can be translated and latched into place with a manual probe. Guided by the preset structure, the shuttle is also repositioned.

\section{EXPERIMENTAL RESULTS}

Finally, we tested the robot motors and legs. Motors were actuated using a programmable Atmel AVR microcontroller whose I/O pins interfaced to an Analog Devices AD8600 16 Channel DAC. Amplifiers on the channels converted the $5 \mathrm{~V}$ maximum outputs of the DAC to the $30 \mathrm{~V}$ signals needed for the motors. The programmability of the test setup allowed us to troubleshoot the inchworm motors and characterize the speed of individual steps of the inchworms.

Each motor we tested measured $700 \mu \mathrm{m} \times 4 \mathrm{~mm}$ in area. A stand-alone motor actuated reliably over $300 \mu \mathrm{m}$ of displacement at speeds up to $200 \mu \mathrm{m} / \mathrm{s}$, an almost $4 \mathrm{x}$ improvement in travel from [5]. A simple flexural spring attached to the shuttle exhibited a calculated reaction force of $45 \mu \mathrm{N}$ under maximum extension. The motor's maximum range was limited by physical stops on the shuttle itself, not by the output 


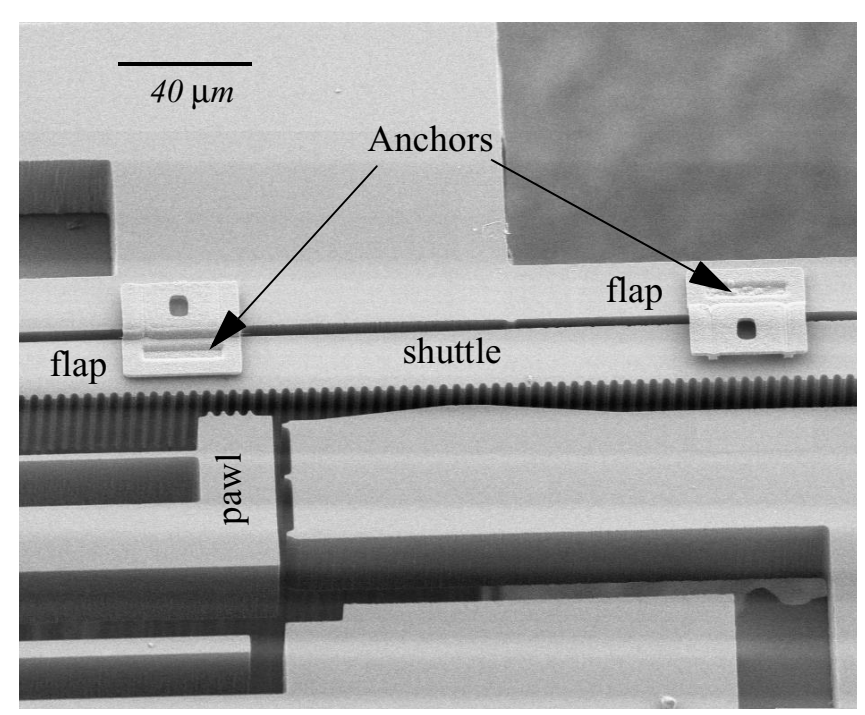

Figure 8. The inchworm motor shuttle and pawl. The flaps are used to constrain the motion of the shuttle. Flap anchors are labeled to show the direction of the flap constraint.

force of the motors. The motor was calculated to maintain a force of at least $120 \mu \mathrm{N}$ at $30 \mathrm{~V}$.

To test the leg, we had to preset the shuttle by $150 \mu \mathrm{m}$ using the latch structure in Figure 9. This gave the leg an initial starting angle of $34^{\circ}$ relative to the plane of the motor (Figure 10).

An additional complication arose, however, because of a design flaw in the motors. Gap stops used to prevent electrical shorting of the GCAs were drawn too small. The resulting fabricated motors exhibited shorting of the gap fingers when the clutch was actuated.

This limited the range of speeds the motor could operate at from $8 \mu \mathrm{m} / \mathrm{s}$ to $32 \mu \mathrm{m} / \mathrm{s}$. Nevertheless, we were able to actuate the robot leg from $34^{\circ}$ to $63^{\circ}$ with a shuttle sweep range of $250 \mu \mathrm{m}$ (Figure 11). Future designs with larger gap stops will prevent electrical shorting and yield higher performance leg actuation.

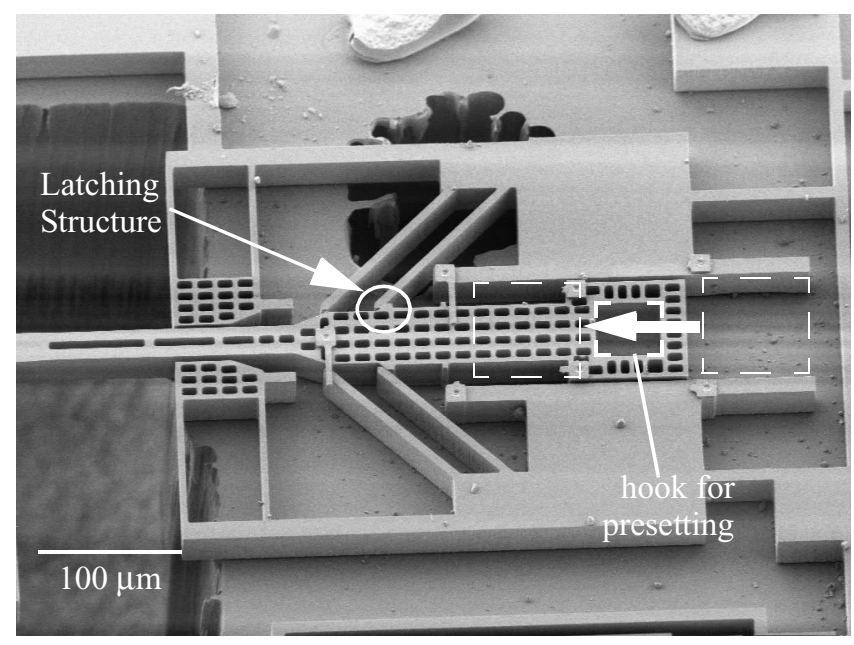

Figure 9. SEM of latch structure midway between starting position and latched position. Probe tip is used to move structure into place.

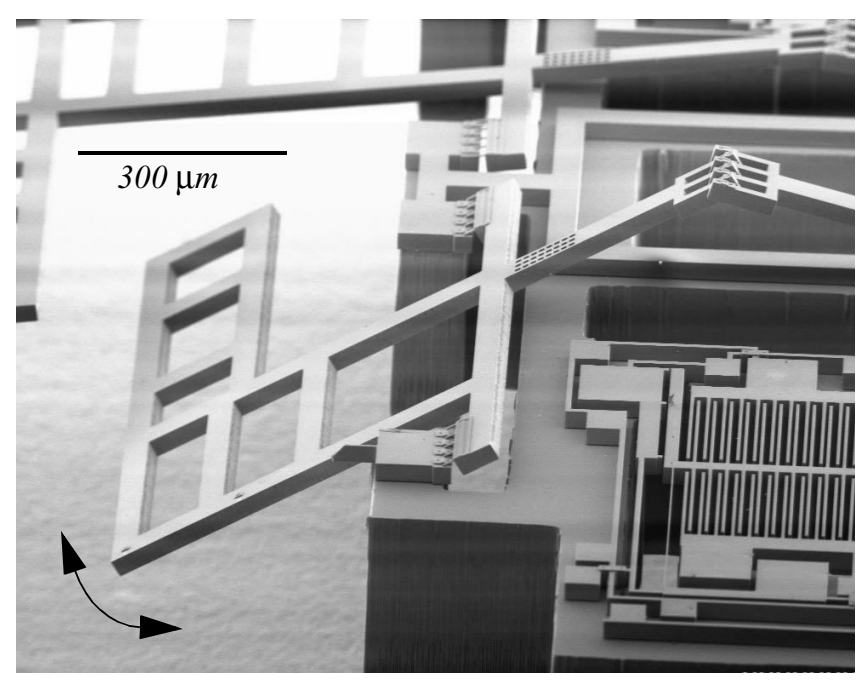

Figure 10. SEM of robot leg in preset condition. Leg was actuated through almost $30^{\circ}$ of angular deflection

By adding polysilicon flaps to constrain the motor shuttle, we introduced a possible source of adhesion and friction to the motor design. Yeh demonstrated in friction tests of polysilicon on nitride that the force required to slide a shuttle along the substrate and rotate an unloaded hinge required between 1$43 \mu \mathrm{N}$, values much greater than the weight of the lever arm (3.2 nN) [8].

To test the amount of static friction an inchworm actuator must overcome to move the shuttle, we designed simple SOI sliding test structures attached to polysilicon vernier springs (Figure 12). The test structures were designed both with and without substrate underneath. This allowed us to compare sliding polysilicon flaps along SOI to sliding an SOI beam along the silicon substrate.

A histogram of the force required to overcome static friction and adhesion in each of the test structures is shown in Figure 13. Force required to move the SOI beam ranged from

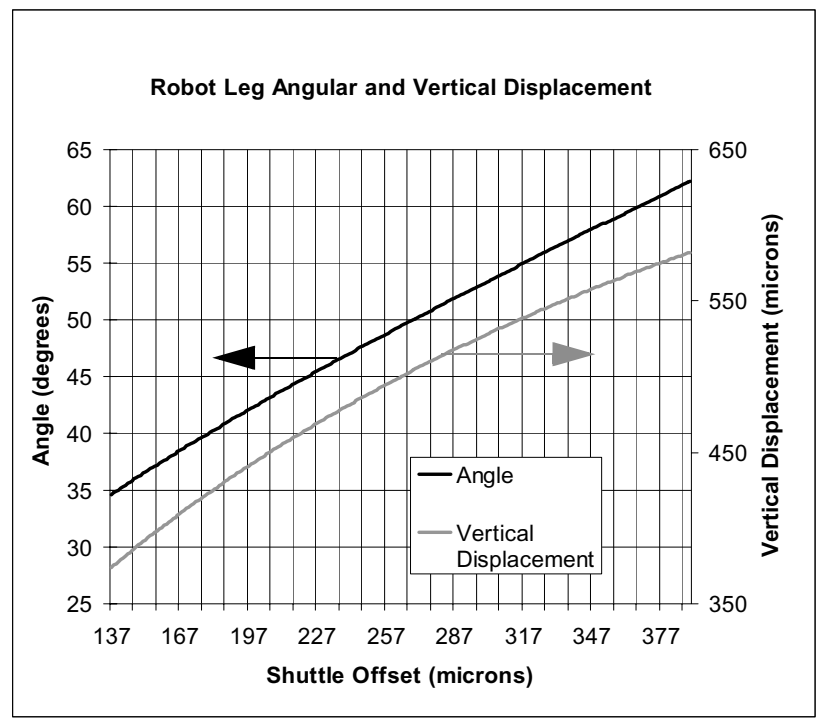

Figure 11. Angular deflection and vertical displacement of leg tip as a function of shuttle travel of inchworm motor. Shuttle actuated over $250 \mu \mathrm{m}$ in this example. 


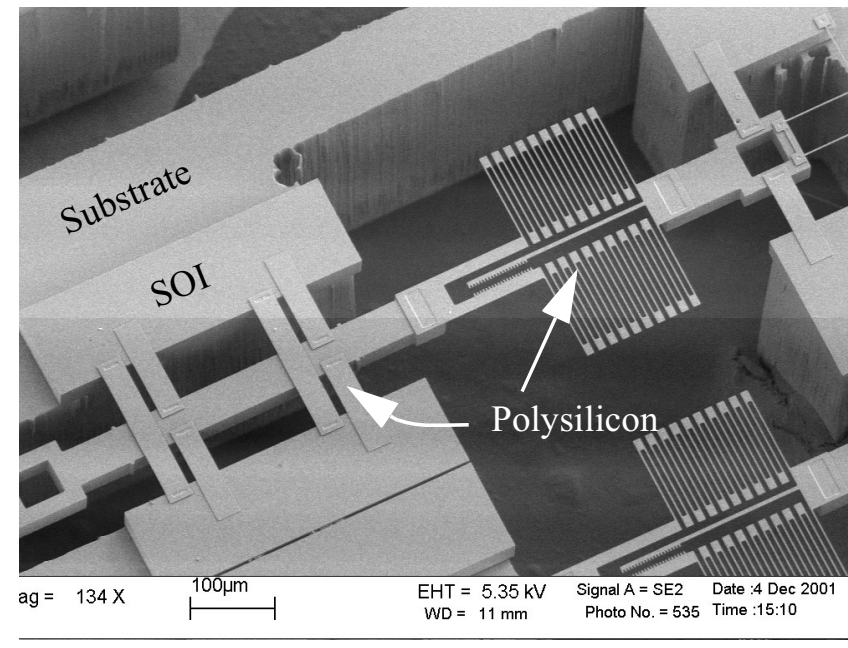

Figure 12. SEM of the sliding test structure. A vernier spring was used to measure the force required to move polysilicon flaps over the SOI device layer.

$0.5-13 \mu \mathrm{N}$ with substrate and $0.5-15 \mu \mathrm{N}$ without substrate. Peak value recurrence for both sliding structures was between 1-3 $\mu \mathrm{N}$.

It is important to note that there are several data points not included in this graph. After storing the test structures in normal laboratory conditions for approximately one month, sliders with polysilicon flaps required forces much greater than the vernier springs could measure $(>>25 \mu \mathrm{N})$. This was probably due to adhesive forces from ambient moisture and will likely need to be addressed in future robot design and testing.

These frictional forces were included in the overall robot model. In order to provide enough force to lift the robot, we designed the inchworm actuators to output a calculated 120 $\mu \mathrm{N}$ of force. This figure takes into account the approximate weight of the robot $(\sim 70 \mu \mathrm{N})$, the mechanical advantage of the linkages $(\sim 1)$, and the maximum possible frictional and adhesive forces of the flaps and hinges $(\sim 20 \mu \mathrm{N})$.

\section{CONCLUSIONS AND FUTURE WORK}

Autonomous microrobots are quickly becoming a reality. One of the last steps is combining a robot leg with effi-

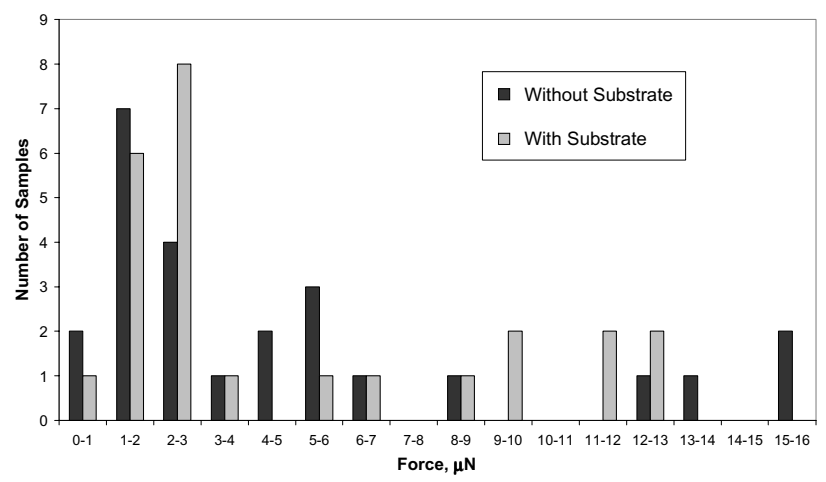

Figure 13. Force measurements taken from the sliding friction test structures in Figure 12. A mean force of $4.6 \mu N$ and median of $2.5 \mu N$ were the same for sliders with and without a substrate. cient, high force actuators. Our process seeks to bridge the gap by integrating electrostatic SOI actuators with high compliance polysilicon structures. The enabling step of the process is planarization of the SOI device layer via reflowed glass. The process allows key improvements to the electrostatic inchworm actuators, including a backside etch underneath the actuators, polysilicon flaps to constrain the shuttle motion, complex wiring, and the ability to preset structures to increase mechanical advantage out-of-plane. Different robot legs, including those with torsional hinges and 2 DOF legs are also being designed for future experiments. Ultimately, we intend to combine legs and actuators from this process with solar cells and a digital CMOS chip to form a complete autonomous microrobot.

It should be noted, however, that this process can be used for more than just designing walking robots. Microtweezers, microoptical scanning systems, and micromanipulators are just a few promising candidates for this process.

\section{ACKNOWLEDGEMENTS}

Chris Keller from MEMS Precision Instruments (http:// www.memspi.com), UC Berkeley Microlab, especially Bob Hamilton and Joe Donnelly, James Wu from Lawrence Livermore Labs, Dimitry Kousminov from Accurel Systems. Research sponsored by DARPA/ITO SDR.

\section{REFERENCES}

[1] N. Miki, I. Shimoyama, "Flight performance of micro-wings rotating in an alternating magnetic field", Technical Digest of MEMS 99, Orlando, FL, (1999), pp. 153-8.

[2] T. Yasuda, I Shimoyama, and H. Miura, "Microrobot locomotion in a mechanical vibration field", Advanced Robotics, 9, 2 (1995).

[3] P.E. Kladitis, V.M. Bright, "Prototype microrobots for micro-positioning and micro-unmanned vehicles", Sensors and Actuators A: Physical, 80 (2000), pp.132-7.

[4] T. Ebefors, J. Mattsson, E. Kalvesten, and G. Stemme, "A micromotion system based on polyimide joint actuators", Eurosensors XII (1998), pp. 391-4.

[5] R. Yeh, S. Hollar, and K.S.J. Pister, "A Single Mask, Large Force, and Large Displacement Electrostatic Inch-worm Motor", Technical Digest of MEMS 01, Interlaken, Switzerland (2001), pp. 260-4.

[6] R. Yeh, E. Kruglick, and K.S.J Pister, "SurfaceMicromachined Components for Articulated Microrobots", J. Microelectromechanical Systems, 5, 1 (1996), pp. 10-17.

[7] A. Yasseen, J. Cawley, and M. Mehregany, "Thick Glass Film Technology for Polysilicon Surface Micromachining," J. Microelectromechanical Systems, 8, 2 (1999), pp. 172-9.

[8] R. Yeh and K.S.J. Pister, "Measurement of Static Friction in Mechanical Couplings of articulated microrobots", Proceedings of SPIE, Austin, TX (1995), pp. 40-50. 